\title{
Pien Tze Huang inhibits the proliferation, and induces the apoptosis and differentiation of colorectal cancer stem cells via suppression of the Notch1 pathway
}

\author{
FEI QI ${ }^{1}$, LIHUI WEI ${ }^{1,3}$, ALING SHEN $^{1}$, YOUQIN CHEN ${ }^{1}$, JIUMAO LIN $^{1,2}$, \\ JIANFENG CHU ${ }^{1}$, QIAOYAN CAI ${ }^{1}$, JIE PAN $^{3}$ and JUN PENG ${ }^{1,2}$ \\ ${ }^{1}$ Academy of Integrative Medicine and ${ }^{2}$ Fujian Key Laboratory of Integrative Medicine on Geriatrics, Fujian University of \\ Traditional Chinese Medicine, Fuzhou, Fujian 350122; ${ }^{3}$ Zhangzhou Pien Tze Huang \\ Pharmaceutical Co., Ltd., Shangjie, Zhangzhou, Fujian 363000, P.R. China
}

Received August 14, 2015; Accepted October 12, 2015

DOI: $10.3892 /$ or.2015.4378

\begin{abstract}
Cancer stem cells (CSCs) possess properties of continuous self-renewal, multi-directional differentiation and natural chemoresistance, leading to the initiation, progression and relapse of cancer. The characteristics of CSCs are strongly associated with multiple cellular pathways such as Notch1 signaling. Therefore, targeting CSCs via suppressing the Notch1 pathway might represent a promising strategy for cancer treatment. The well-known traditional Chinese medicine (TCM) formula Pien Tze Huang (PZH) has long been used as an alternative remedy for various cancers including colorectal cancer (CRC). We previously reported that $\mathrm{PZH}$ contains a broad range of anticancer activities including an inhibitory effect on CSCs. To further elucidate the mode of action of $\mathrm{PZH}$, in this study we isolated the stem-like side population (SP) from the human CRC SW480 cell line to investigate its effect on CSCs as well as the possible molecular mechanisms. As compared with non-SP cells, the isolated SW480 SP cells displayed stronger capacities of spheroid formation in vitro and tumorigenicity in vivo, demonstrating the stem cell-like features of SP cells. However, PZH treatment significantly decreased the percentage of SP cells in a
\end{abstract}

Correspondence to: Dr Jie Pan, Zhangzhou Pien Tze Huang Pharmaceutical Co., Ltd., Shangjie, Zhangzhou, Fujian 363000, P.R. China

E-mail: 13906953017@163.com

Dr Jun Peng, Academy of Integrative Medicine, Fujian University of Traditional Chinese Medicine, 1 Qiuyang Road, Minhou Shangjie, Fuzhou, Fujian 350122, P.R. China

E-mail: pjunlab@hotmail.com

Abbreviations: CRC, colorectal cancer; PZH, Pien Tze Huang; TCM, traditional Chinese medicine; CSC, cancer stem cell; SP, side population

Key words: Pien Tze Huang, traditional Chinese medicine, cancer stem cells, side population, Notch1 pathway dose-dependent manner. In addition, PZH significantly and does-dependently inhibited the viability and promoted the apoptosis and differentiation of the isolated SW480 SP cells. Moreover, $\mathrm{PZH}$ treatment profoundly reduced the mRNA and protein expression of Notch1 and Hes1 in the SP cells. Our findings suggest that PZH negatively modulates the characteristics of CSCs through suppression of the Notch1 signaling pathway.

\section{Introduction}

Increasing evidence indicates that a small population of cancer stem cells (CSCs) exists in many solid tumors including colorectal cancer (CRC) (1-3). CSCs possess the capacities for continuous self-renewal, multi-lineage differentiation and natural resistance to conventional therapies, resulting in cancer relapse and metastasis and eventually the failure of clinical anticancer treatment $(4,5)$. The characteristics of CSCs are highly regulated by multiple cellular signaling cascades including the Notch, Wnt and Hedgehog pathways (6-8). The Notch signaling pathway is a fundamental signaling system essential for embryonic development and tissue homeostasis $(9,10)$. Activation of Notch signaling is triggered by the interaction of a family of ligands with their specific transmembrane-spanning Notch receptors (11). In mammals, five ligands (Jagged1, Jagged2, Delta1, Delta2 and Delta3) and four Notch proteins (Notch1, 2, 3 and 4) have been identified, of which Notch1 is the best studied $(12,13)$. Upon ligand binding, Notch protein is cleaved by proteolytic processing to release an intracellular domain of Notch (NICD) from the membrane into the cytoplasm. NICD in turn translocates into the nucleus to participate in the transcriptional regulation of target genes mediating many biological processes such as cell differentiation, proliferation and apoptosis $(14,15)$. Previous studies have proposed that aberrant activation of the Notch pathway is tightly associated with the development of various malignancies including CRC (16-18). Moreover, Notch1 has been shown to be involved in the control of CSCs by regulating the expression of downstream gene Hes1; and targeting Notch1 could result in the decrease in CSC proliferation (19-22). 
Therefore, attacking CSCs via suppressing the Notch pathway may provide an opportunity to improve the efficacy of conventional cancer therapies $(6,7,23)$.

Traditional Chinese medicine (TCM) has been used for thousands of years in China to clinically treat various diseases including cancer. Recently, TCM has attracted great interest in the fields of stem cell biology since various naturally occurring components have been shown to possess anti-CSC activities (24). Pien Tze Huang (PZH), a well-known TCM formula prescribed in the Chinese Ming Dynasty more than 450 years ago, has been used in China and Southeast Asia for centuries as a folk remedy for cancers. Previously, we demonstrated that PZH possesses a broad range of anticancer activities through affecting multiple intracellular targets (25-36). More interestingly, we recently reported that $\mathrm{PZH}$ inhibits the growth of CSCs isolated from colon carcinoma HT-29 cells (37). To further assess the anti-CSC activity of PZH and explore the underlying mechanism, in the present study we isolated stem-like side population (SP) from the human colorectal cancer SW480 cell line, and evaluated the effect of PZH on the proliferation, apoptosis and differentiation of isolated SW480 SP cells as well as on the activation of the Notch1 signaling pathway.

\section{Materials and methods}

Materials and reagents. Leibovitz's L-15 medium, serum-free stem cell culture medium (DMEM)/F12, fetal bovine serum (FBS), B27 supplement (50X), penicillin-streptomycin, trypsin-EDTA and StemPro Accutase Cell Dissociation Reagent were purchased from Life Technologies Corp. (Grand Island, NY, USA). EGF and bFGF were obtained from Peprotech (Rocky Hill, NJ, USA). Hoechst 33342 and verapamil were purchased from Sigma Chemicals (St. Louis, MO, USA). Antibodies against Notch1, Hes1, CK20 and GAPDH were purchased from Abcam (Hong Kong). Horseradish peroxidase (HRP)-conjugated secondary antibodies were obtained from Cell Signaling Technology (Beverly, MA, USA). BCA protein assay reagent kit and Super-Signal West Pico Chemiluminescent Substrate were obtained from Pierce (Rockford, IL, USA). TRIzol reagent, PrimeScript RT reagent kit and SYBR Premix Ex Taq II kit were provided by Takara Biotechnology Co., Ltd. (Dalian, Liaoning, China).

Preparation of PZH. PZH was obtained from and authenticated by Zhangzhou Pien Tze Huang Pharmaceutical Co., Ltd., China (Chinese FDA approval no. Z35020242). The stock solution of PZH was freshly prepared by dissolving the powder in phosphate-buffered saline (PBS) to a concentration of $20 \mathrm{mg} / \mathrm{ml}$. Working concentrations of PZH were made by diluting the stock solution with culture medium.

Cell culture. The human colorectal cancer cell line SW480 was purchased from the Cell Bank of the Chinese Academy of Sciences (Shanghai, China). Cells were cultured in Leibovitz's L-15 medium, containing $10 \%$ (v/v) FBS, $100 \mathrm{U} / \mathrm{ml}$ penicillin and $100 \mu \mathrm{g} / \mathrm{ml}$ streptomycin in a $37^{\circ} \mathrm{C}$ humidified incubator with $5 \% \mathrm{CO}_{2}$.

Isolation and culture of side population (SP) cells. SP cells from SW480 cells were isolated and analyzed using Moflo
XDP cell sorter flow cytometry (Beckman Coulter, Fullerton, CA, USA) as previously described (37). Excitation of Hoechst dye was performed using a UV laser at $355 \mathrm{~nm}$, and the fluorescence was measured with a $450 \pm 25 \mathrm{~nm}$ filter (Hoechst blue) and a $620 \pm 15 \mathrm{~nm}$ filter (Hoechst red). Sorted SP cells were cultured in DMEM/F12, containing B27 (1X), $20 \mathrm{ng} / \mathrm{ml}$ EGF and $20 \mathrm{ng} / \mathrm{ml} \mathrm{bFGF}$.

In vitro sphere formation assay. SP cells were seeded at a density of 250 cells/well in 24-well ultra-low attachment plates (Corning, Lowell, MA, USA) and grown in DMEM/F12 serum-free stem cell culture medium. The medium was added every 2 days. After 7 days the spheroids were photographed and quantified. A spheroid with a diameter $>100 \mu \mathrm{m}$ was considered to be a full sphere.

In vivo tumorigenic assay. Athymic female $\mathrm{BABL} / \mathrm{c}$ nude mice were purchased from Shanghai SLAC Laboratory Animal Co., Ltd. (Shanghai, China). Mice were housed under pathogen-free conditions with a 12-h light/dark cycle. Food and water were given ad libitum. Freshly sorted 500 SP or non-SP cells were resuspended in $50 \mu \mathrm{l} \mathrm{PBS}$ and then mixed with $50 \mu 1$ Matrigel (BD Biosciences, San Jose, CA, USA). SP or non-SP cells were respectively injected into the right or left subaxillary area of the mice. Tumor development was monitored every 2 days for 7 weeks. All animal treatments were strictly in accordance with the International Ethics Guidelines and the National Institutes of Health Guide concerning the Care and Use of Laboratory Animals, and the experiments were approved by the Institutional Animal Care and Use Committee of Fujian University of Traditional Chinese Medicine.

Evaluation of cell viability by MTS assay. Sorted SW480 SP cells were seeded into 96-well plates at a density of $1.0 \times 10^{4}$ cells/well in serum-free stem cell culture medium DMEM/F12. After cells were treated with various concentrations of $\mathrm{PZH}$ for $48 \mathrm{~h}, 20 \mu \mathrm{l}$ MTS was added to each well, and the samples were incubated for an additional $2 \mathrm{~h}$ at $37^{\circ} \mathrm{C}$. The absorbance was measured at $490 \mathrm{~nm}$ using an ELISA reader (Model ELX800; BioTek Instruments, Inc., Winooski, VT, USA).

Apoptosis analysis with Hoechst staining. SP cells were seeded into 24-well plates at a density of $1 \times 10^{5}$ cells/well in serum-free stem cell culture medium DMEM/F12. After treatment with various concentrations of PZH for $48 \mathrm{~h}$, the cells were dissociated by StemPro Accutase Cell Dissociation Reagent and stained with Hoechst $33342(1 \mu \mathrm{g} / \mathrm{ml})$ for $10 \mathrm{~min}$. Cell apoptosis was observed under a fluorescence microscope and photographed at a magnification of $\mathrm{x} 400$.

RNA extraction and qPCR analysis. SP cells were seeded into 24-well plates at a density of $1 \times 10^{5}$ cells/well in serum-free stem cell culture medium DMEM/F12. After cells were treated with various concentrations of PZH for $48 \mathrm{~h}$, total RNA was isolated with TRIzol reagent and reverse-transcribed with PrimeScript RT reagent kit according to the manufacturer's instructions. The obtained cDNA was used to determine the expression of Notch1, Hes1 and CK20 by qPCR. $\beta$-actin was used as internal control. Primer sequences used in PCR are listed in Table I. 

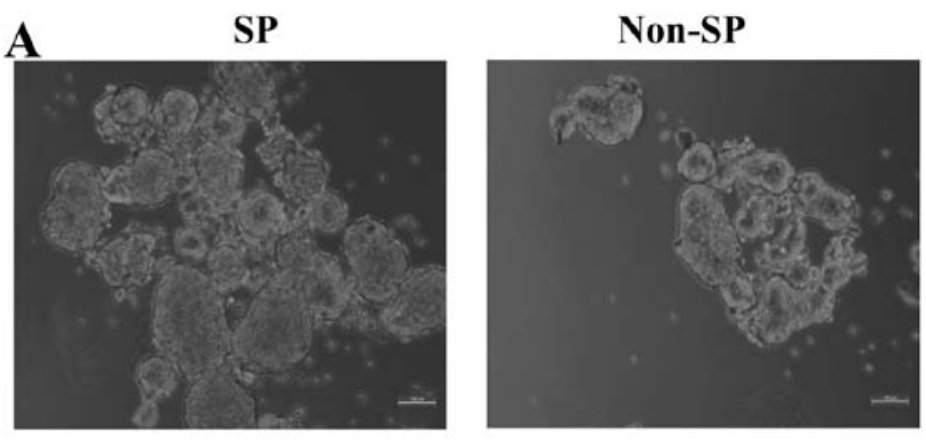

B

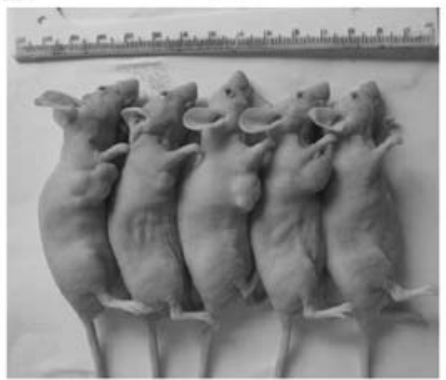

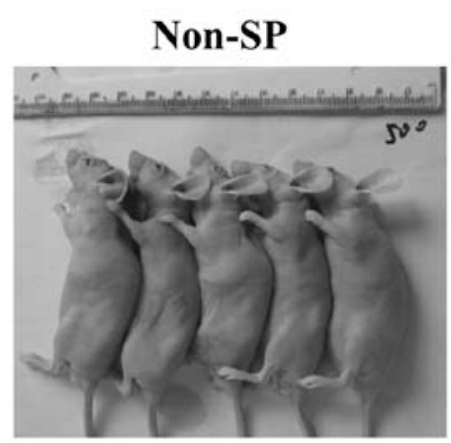

Figure 1. Side population (SP) cells sorted from the SW480 cell line exhibited high tumorigenic capacity in vivo and in vitro. (A) SP cells were grown in serum-free stem cell culture medium for 7 days. A spheroid with a diameter $>100 \mu \mathrm{m}$ was considered to be a full sphere. Images are representatives and data are shown as averages with SD from three independent experiments. ${ }^{*} \mathrm{P}<0.05$, vs. the untreated control cells. (B) Freshly sorted 500 SP or non-SP cells were respectively injected into the right or left subaxillary area of the nude mice. Seven weeks later tumor development was evaluated.

Table I. Primer sequences for PCR.

\begin{tabular}{ll}
\hline Gene & \multicolumn{1}{c}{ Primers $\left(5^{\prime} \rightarrow 3^{\prime}\right)$} \\
\hline CK20 & F: CGAGTTGCTTCCCGATACTTC \\
& R: ACTTGCCAGCATACAACCCAA \\
Notch1 & F: GGTGCCGAACCAATACAACCCTCT \\
& R: TTGCTGCTGCTGGATGTTTGCTG \\
Hes1 & F: CTGGAGAGGCGGCTAAGGTGTT \\
& R: TGTTGCTGGTGTAGACGGGGAT \\
$\beta$-actin & F: AAGGTGACAGCAGTCGGTTGGAG \\
& R: GAGAAGTGGGGTGGCTTTTAGGAT
\end{tabular}

F, forward; R, reverse.

Western blot analysis. SP cells were seeded in 6-well plates at a density of $4 \times 10^{5}$ cells/well in serum-free stem cell culture medium DMEM/F12 and treated with various concentrations of $\mathrm{PZH}$ for $48 \mathrm{~h}$. The treated cells were lysed with mammalian cell lysis buffer containing protease and phosphatase inhibitor cocktails. Total protein concentrations were determined by BCA assay. A total of $30 \mu \mathrm{g}$ of total proteins was resolved on $10 \%$ SDS-PAGE gels and electroblotted. The PVDF membranes were blocked with blocking buffer and probed with primary antibodies against Notch1, Hes1, CK20 or GAPDH $(1: 1,000)$ overnight at $4^{\circ} \mathrm{C}$ and subsequently incubated with the appropriate HRP-conjugated secondary antibody $(1: 5,000)$ followed by Super-Signal West Pico chemiluminescence detection.
Statistical analysis. All data are presented as the averages of three determinations and were analyzed using the SPSS package for Windows (version 18.0). Statistical analysis of the data was performed with Student's t-test and ANOVA.

\section{Results}

SP cells sorted from the SW480 cell line exhibit high tumorigenic capacity in vivo and in vitro. Spheroid formation and tumorigenicity assays were performed to assess the tumorigenic capacity of SP and non-SP in vitro and in vivo. The numbers of tumor spheroids formed from the SP and non-SP cells were $12 \pm 1.6$ and $3 \pm 1$, respectively $(\mathrm{P}<0.05$ vs. the SP group), suggesting that the SP cells exhibited higher tumor spheroid formation capacity when compared with the non-SP cells. (Fig. 1A). Moreover, in the in vivo study, SP cells initiated tumors with 500 cells in 4 of 5 mice $(80 \%)$, whereas non-SP cells failed to form tumors ( 0 of 5 mice) ( $0 \%$ ) (Fig. 1B), indicating that SP cells displayed significantly stronger tumorigenicity in vivo, demonstrating that the isolated SW480 SP cells possessed characteristics similar to cancer stem cells.

PZH decreases the percentage of SP cells in the SW480 cells. To evaluate the effect of PZH on cancer stem cells, we determined the percentage of SP cells. Similar to the positive control verapamil, PZH significantly and dose-dependently decreased the SP proportion from $0.74 \pm 0.05$ to $0.08 \pm 0.02 \%$ in the SW480 cells ( $\mathrm{P}<0.05$ vs. the control group) (Fig. 2).

PZH inhibits the proliferation of the sorted SW480 SP cells. In order to assess the effect of PZH on the proliferation of CSCs, 

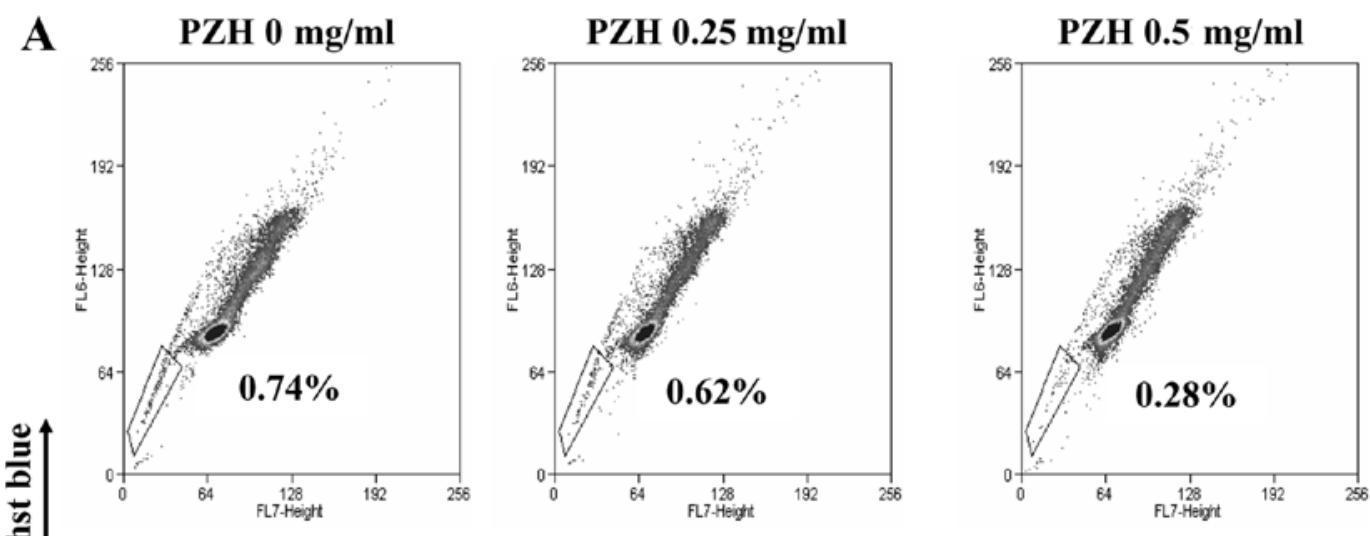

PZH $0.75 \mathrm{mg} / \mathrm{ml}$
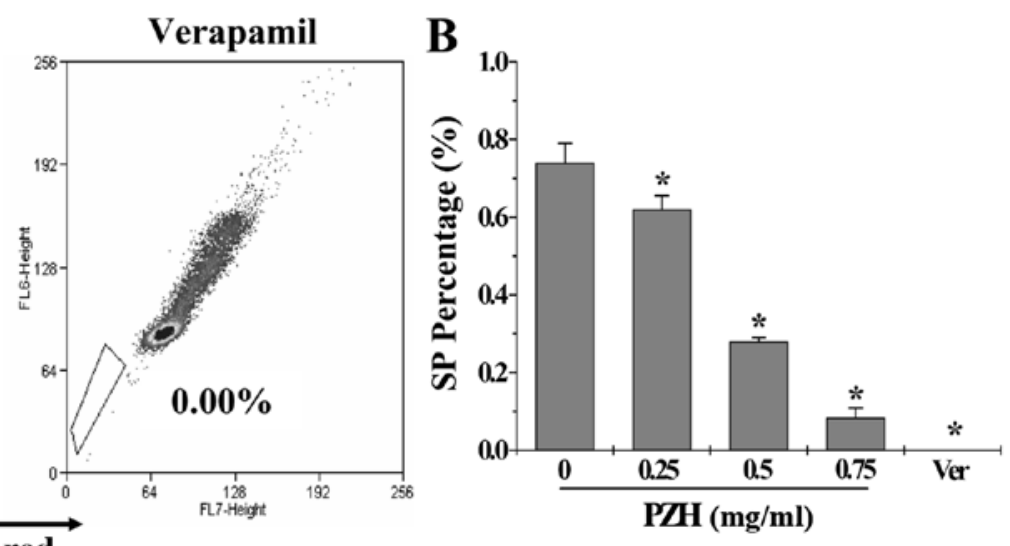

Figure 2. PZH reduces the percentage of the side population (SP) in SW480 cells. (A) After treatment with various concentrations of PZH for $48 \mathrm{~h}$, SW480 cells were stained with Hoechst 33342 and percentages of SP cells were analyzed by FACS. Verapamil was used as a positive control. (B) Quantification of FACS analysis. Images are representatives and data are expressed as the averages with SD from 3 independent experiments. ${ }^{*} \mathrm{P}<0.05$, vs. untreated control cells.

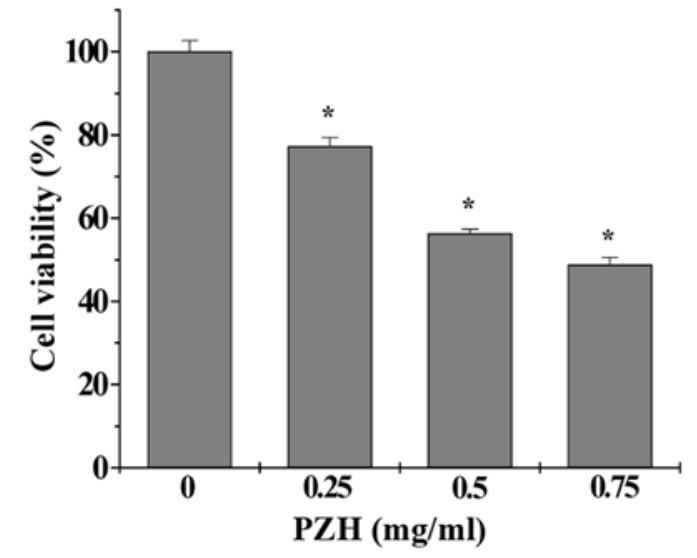

Figure 3. PZH inhibits the viability of isolated SW480 SP cells. After treatment with various concentrations of PZH for $48 \mathrm{~h}$, the viability of the SP cells was determined by MTS assay. Data are averages with SD from three independent experiments. ${ }^{*} \mathrm{P}<0.05$, vs. the untreated control cells.

the viability of the sorted SW480 SP cells was measured by MTS assay. PZH treatment significantly and dose-dependently decreased the SP viability by $22.75-51.16 \%$ compared with the control $(\mathrm{P}<0.05)$ (Fig. 3).

PZH enhances the apoptosis of sorted SW480 SP cells. Cell apoptosis was determined by Hoechst staining. PZH-treated cells displayed typical apoptotic morphological characteris- tics including condensed chromatin and fragmented nuclear morphology, while the untreated cell nuclei were homogeneously stained and less intense (Fig. 4).

PZH promotes differentiation of sorted SW480 SP cells. Differentiation of CSCs was evaluated by the expression of differentiation marker CK20. Data from qPCR and western blot analyses showed that PZH profoundly increased the mRNA and protein expression of CK20 in the SP cells (Fig. 5).

PZH suppresses the Notchl pathway in the sorted SW480 $S P$ cells. The activation of Notch1 signaling was analyzed by examining the expression of key mediators, Notch1 and Hes1. PZH markedly reduced the expression of Notch1 and Hes1 in the isolated SW480 SP cells, at both the transcriptional and translational levels (Fig. 5).

\section{Discussion}

CRC is one of the most common cancers worldwide, accounting for more than one million new cases and over a half million deaths each year $(38,39)$. Chemotherapy is an important modality of CRC treatment. However, drug resistance and cytotoxicity limit the effectiveness of currently used chemotherapies (40). Mounting evidence suggests that the existence of CSCs is one of the major causative factors for cancer initiation and progression, and eventually leading to the failure of clinical treatment. On the other hand, natural products, 


\section{PZH (mg/ml)}

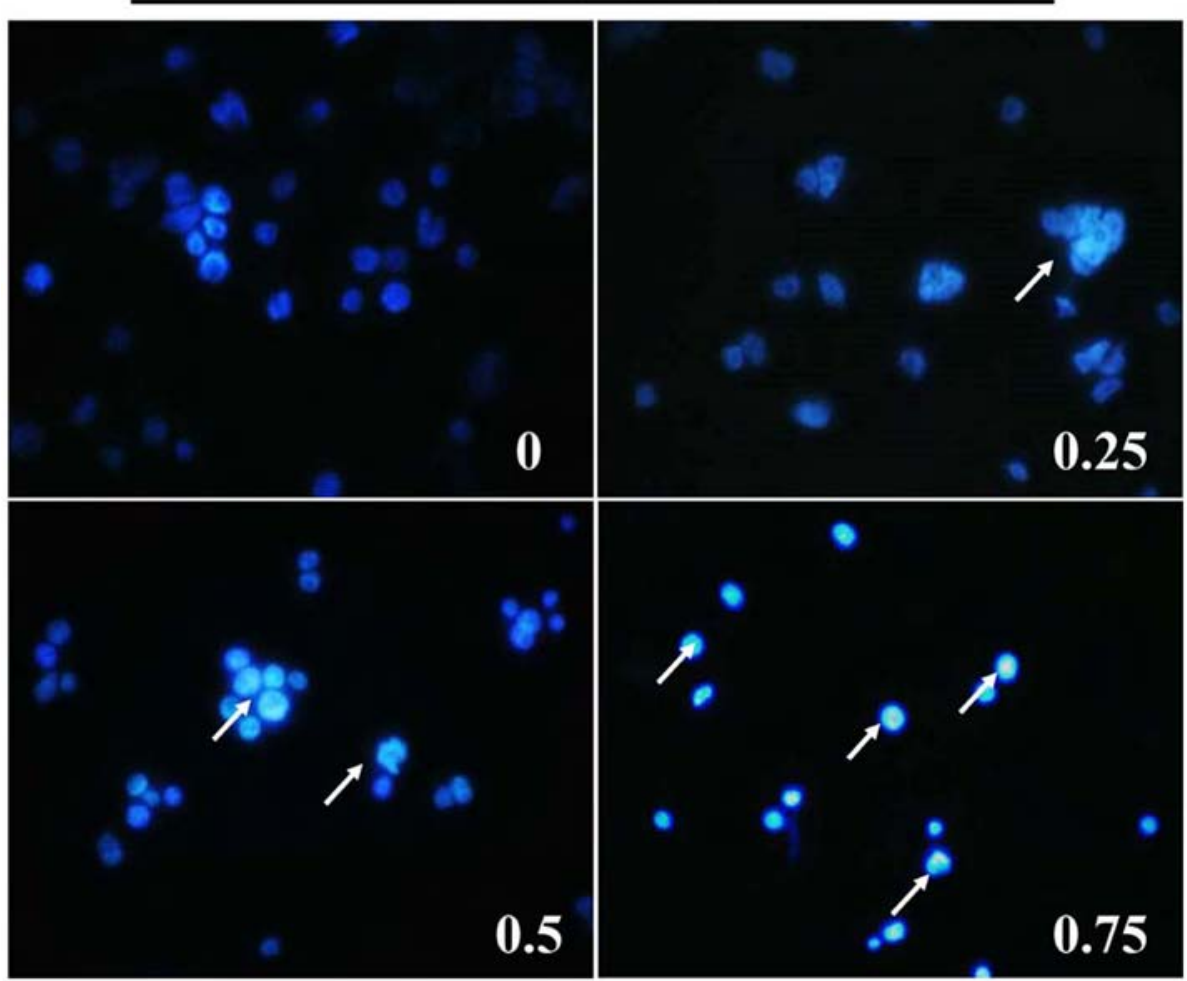

Figure 4. PZH promotes the apoptosis of isolated SW480 SP cells. SP cells were treated with various concentrations of PZH for $48 \mathrm{~h}$. Cell apoptosis was determined by Hoechst staining. Images are representatives of three independent experiments.
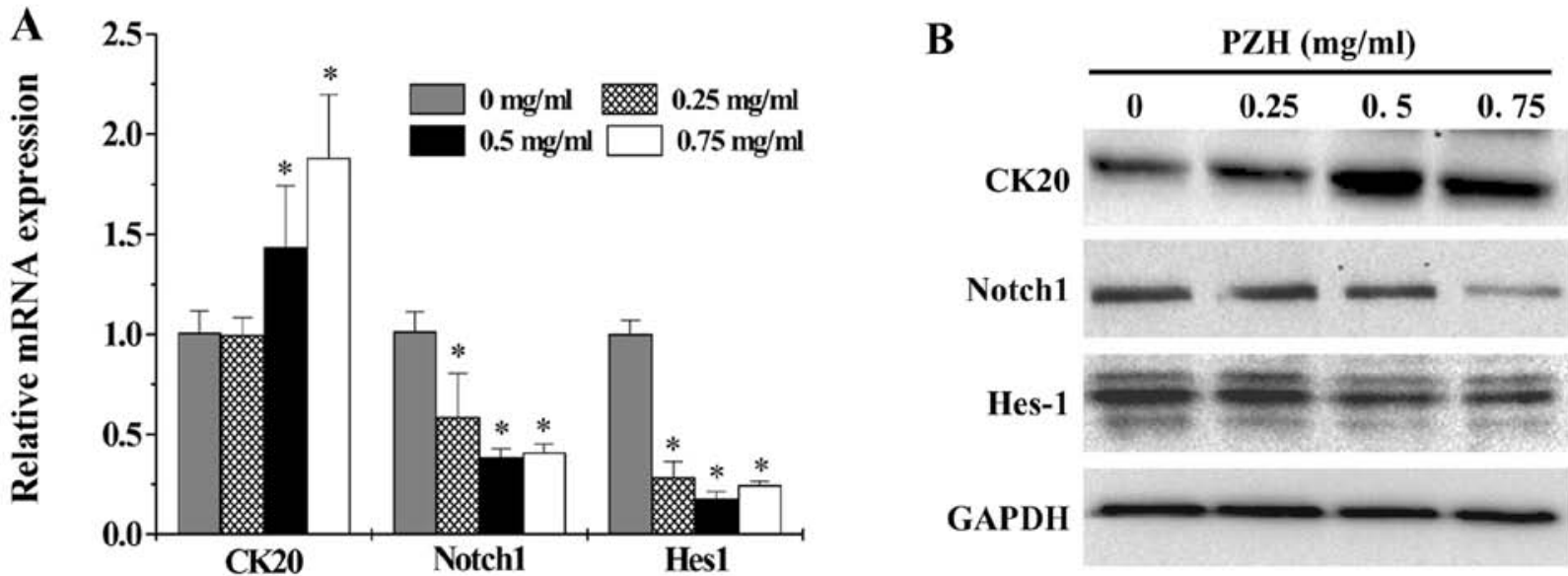

Figure 5. PZH modulates the expression of CD20, Notch1 and Hes1 in the isolated SW480 SP cells. SP cells were treated with various concentrations of PZH for 48 h. (A) The mRNA levels of CK20, Notch1 and Hes1 were determined by qPCR. $\beta$-actin was used as an internal control. The data were normalized to the mean mRNA expression in control cells and represented as the fold of the control. Data are shown as averages with SD from three independent experiments. ${ }^{*} \mathrm{P}<0.05$, vs. the untreated control cells. (B) The protein expression levels of CK20, Notch1 and Hes1 were analyzed by western blot analysis. GAPDH was used as an internal control. Images are representatives of three independent experiments.

particularly TCM, have attracted great attention in the field of cancer research due to their definite therapeutic efficacy and fewer adverse effects. Therefore, discovering naturally occurring agents and/or targeting CSCs have become promising strategies for cancer therapies. The well-known TCM formula PZH has long been used in China to clinically treat various malignancies including CRC. We previously demonstrated that PZH contains a broad range of anticancer activities including an inhibitory effect on CSCs (25-37), suggesting that PZH could be developed as a novel multi-potent therapeutic agent for cancer treatment.

Isolation and identification are critical steps for CSC study. A commonly used approach to isolate stem cells is SP analysis using fluorescence-activated cell sorting (FACS), which is based on the ability of CSCs to expel incorporated Hoechst dyes due to the overexpression of multidrug resistance (MDR) genes and ATP-binding cassette $(\mathrm{ABC})$ transporters $(41,42)$. $\mathrm{SP}$ cells have been identified in various types of cancer and are 
shown to be correlated with tumor grade and patient prognosis. One strategy of functional identification for isolated CSCs is implanting SP cells in immune-deficient mice to assess their in vivo tumor-initiating capacity (43). Another approach to verify the stemness of isolated CSCs is in vitro sphere-forming assay, with a special serum-free culture system where only CSC-enriched cells can survive and form three-dimensional spheres (44). In the present study, we isolated stem-like SP cells from human CRC SW480 cells and confirmed that the sorted SP cells possessed characteristics of CSCs since SP cells displayed stronger capacities of spheroid formation in vitro and tumorigenicity in vivo, as compared with the non-SP cells. More importantly, here we demonstrated that PZH significantly decreased the percentage of SP cells in a dose-dependent manner. Moreover, PZH treatment significantly and dose-dependently inhibited the proliferation, and promoted the apoptosis and differentiation of the isolated SW480 SP cells.

The Notch pathway plays important roles in many biological processes including tissue patterning and morphogenesis, cell differentiation, proliferation and apoptosis, which therefore is essential for embryonic development and tissue homeostasis in adulthood $(9,10)$. Alterations in the components of the Notch pathway have been strongly associated with cancer and appear to be involved in the regulation of CSCs (19-22). Thus, inhibition of the Notch pathway may provide a target for the development of new anti-CSC therapeutic agents. Using qPCR and western blot analyses, we found that PZH treatment markedly suppressed the expression of Notch1 and Hes1 in the isolated SW480 SP cells, two mediators in the Notch pathway.

In conclusion, PZH may negatively modulate the characteristics of CSCs through suppression of the Notch1 signaling pathway.

\section{Acknowledgements}

The present study was sponsored by the National Natural Science Foundation of China (81403390), the Research Fund of the Education Bureau of Fujian Province (JA14162), the Joint-Project of Education and Health Bureaus of Fujian Province (WKJ-FJ-21), and the Developmental Fund of Chen Keji Integrative Medicine (CKJ2013012, CKJ2013013 and CKJ2014004).

\section{References}

1. Clarke MF, Dick JE, Dirks PB, Eaves CJ, Jamieson CH, Jones DL, Visvader J, Weissman IL and Wahl GM: Cancer stem cells - perspectives on current status and future directions: AACR Workshop on cancer stem cells. Cancer Res 66: 9339-9344, 2006.

2. Jordan CT, Guzman ML and Noble M: Cancer stem cells. N Engl J Med 355: 1253-1261, 2006.

3. Dalerba P, Cho RW and Clarke MF: Cancer stem cells: Models and concepts. Annu Rev Med 58: 267-284, 2007.

4. Costea DE, Tsinkalovsky O, Vintermyr OK, Johannessen AC and Mackenzie IC: Cancer stem cells - new and potentially important targets for the therapy of oral squamous cell carcinoma. Oral Dis 12: 443-454, 2006.

5. Locke M, Heywood M, Fawell S and Mackenzie IC: Retention of intrinsic stem cell hierarchies in carcinoma-derived cell lines. Cancer Res 65: 8944-8950, 2005.

6. Takebe N, Harris PJ, Warren RQ and Ivy SP: Targeting cancer stem cells by inhibiting Wnt, Notch, and Hedgehog pathways. Nat Rev Clin Oncol 8: 97-106, 2011.
7. Bolós V, Blanco M, Medina V, Aparicio G, Díaz-Prado S and Grande E: Notch signalling in cancer stem cells. Clin Transl Oncol 11: 11-19, 2009.

8. Liu S, Dontu G, Mantle ID, Patel S, Ahn NS, Jackson KW, Suri $\mathrm{P}$ and Wicha MS: Hedgehog signaling and Bmi-1 regulate self-renewal of normal and malignant human mammary stem cells. Cancer Res 66: 6063-6071, 2006.

9. Noggle SA, Weiler D and Condie BG: Notch signaling is inactive but inducible in human embryonic stem cells. Stem Cells 24: 1646-1653, 2006.

10. Miyamoto S and Rosenberg DW: Role of Notch signaling in colon homeostasis and carcinogenesis. Cancer Sci 102: 1938-1942, 2011.

11. Artavanis-Tsakonas S, Rand MD and Lake RJ: Notch signaling: Cell fate control and signal integration in development. Science 284: 770-776, 1999.

12. Ohishi K, Varnum-Finney B, Flowers D, Anasetti C, Myerson D and Bernstein ID: Monocytes express high amounts of Notch and undergo cytokine specific apoptosis following interaction with the Notch ligand, Delta-1. Blood 95: 2847-2854, 2000.

13. Bolós V, Grego-Bessa J and de la Pompa JL: Notch signaling in development and cancer. Endocr Rev 28: 339-363, 2007.

14. Hansson EM, Lendahl U and Chapman G: Notch signaling in development and disease. Semin Cancer Biol 14: 320-328, 2004.

15. Iso T, Kedes L and Hamamori Y: HES and HERP families: Multiple effectors of the Notch signaling pathway. J Cell Physiol 194: 237-255, 2003.

16. Zhang Y, Li B, Ji ZZ and Zheng PS: Notch1 regulates the growth of human colon cancers. Cancer 116: 5207-5218, 2010.

17. Reedijk M, Odorcic S, Zhang H, Chetty R, Tennert C, Dickson BC, Lockwood G, Gallinger S and Egan SE: Activation of Notch signaling in human colon adenocarcinoma. Int $\mathrm{J}$ Oncol 33: 1223-1229, 2008.

18. Miele L, Golde T and Osborne B: Notch signaling in cancer. Curr Mol Med 6: 905-918, 2006.

19. Qiu M, Peng Q, Jiang I, Carroll C, Han G, Rymer I, Lippincott J, Zachwieja J, Gajiwala K, Kraynov E, et al: Specific inhibition of Notch1 signaling enhances the antitumor efficacy of chemotherapy in triple negative breast cancer through reduction of cancer stem cells. Cancer Lett 328: 261-270, 2013.

20. Sharma A, Paranjape AN, Rangarajan A and Dighe RR: A monoclonal antibody against human Notch1 ligand-binding domain depletes subpopulation of putative breast cancer stem-like cells. Mol Cancer Ther 11: 77-86, 2012.

21. Wang J, Wang C, Meng Q, Li S, Sun X, Bo Y and Yao W: siRNA targeting Notch-1 decreases glioma stem cell proliferation and tumor growth. Mol Biol Rep 39: 2497-2503, 2012.

22. Fre S, Huyghe M, Mourikis P, Robine S, Louvard D and Artavanis-Tsakonas S: Notch signals control the fate of immature progenitor cells in the intestine. Nature 435: 964-968, 2005.

23. Ward RJ and Dirks PB: Cancer stem cells: At the headwaters of tumor development. Annu Rev Pathol 2: 175-189, 2007.

24. Efferth T: Stem cells, cancer stem-like cells, and natural products. Planta Med 78: 935-942, 2012.

25. Lin JM, Wei LH, Chen YQ, Liu XX, Hong ZF, Sferra TJ and Peng J: Pien Tze Huang induced apoptosis in human colon cancer HT-29 cells is associated with regulation of the Bcl-2 family and activation of caspase 3. Chin J Integr Med 17: 685-690, 2011.

26. Zhuang Q, Hong F, Shen A, Zheng L, Zeng J, Lin W, Chen Y, Sferra TJ, Hong $Z$ and Peng J: Pien Tze Huang inhibits tumor cell proliferation and promotes apoptosis via suppressing the STAT3 pathway in a colorectal cancer mouse model. Int J Oncol 40: 1569-1574, 2012.

27. Shen AL, Hong F, Liu LY, Lin JM, Zhuang QC, Hong ZF and Peng J: Effects of Pien Tze Huang on angiogenesis in vivo and in vitro. Chin J Integr Med 18: 431-436, 2012.

28. Shen A, Hong F, Liu L, Lin J, Wei L, Cai Q, Hong Z and Peng J: Pien Tze Huang inhibits the proliferation of human colon carcinoma cells by arresting G1/S cell cycle progression. Oncol Lett 4: 767-770, 2012.

29. Shen A, Chen Y, Hong F, Lin J, Wei L, Hong Z, Sferra TJ and Peng J: Pien Tze Huang suppresses IL-6-inducible STAT3 activation in human colon carcinoma cells through induction of SOCS3. Oncol Rep 28: 2125-2130, 2012.

30. Shen A, Lin J, Chen Y, Lin W, Liu L, Hong Z, Sferra TJ and Peng J: Pien Tze Huang inhibits tumor angiogenesis in a mouse model of colorectal cancer via suppression of multiple cellular pathways. Oncol Rep 30: 1701-1706, 2013.

31. Huang M, Zhao H, Xu W, Chu K, Hong Z, Peng J and Chen L: Rapid simultaneous determination of twelve major components in Pien Tze Huang by ultra-performance liquid chromatography coupled with triple quadrupole mass spectrometry. J Sep Sci 36: 3866-3873, 2013. 
32. Chen H, Shen A, Zhang Y, Chen Y, Lin J, Lin W, Sferra T and Peng J: Pien Tze Huang inhibits hypoxia-induced epithelial-mesenchymal transition in human colon carcinoma cells through suppression of the HIF-1 pathway. Exp Ther Med 7: $1237-1242,2014$

33. Shen A, Chen H, Chen Y, Lin J, Lin W, Liu L, Sferra TJ and Peng J: Pien Tze Huang overcomes multidrug resistance and epithelial-mesenchymal transition in human colorectal carcinoma cells via suppression of TGF- $\beta$ pathway. Evid Based Complement Alternat Med 2014: 679436, 2014.

34. Lin W, Zhuang Q, Zheng L, Cao Z, Shen A, Li Q, Fu C, Feng J and Peng J: Pien Tze Huang inhibits liver metastasis by targeting TGF- $\beta$ signaling in an orthotopic model of colorectal cancer. Oncol Rep 33: 1922-1928, 2015.

35. Chen H, Feng J, Zhang Y, Shen A, Chen Y, Lin J, Lin W, Sferra TJ and Peng J: Pien Tze Huang inhibits hypoxia-induced angiogenesis via HIF-1 $\alpha$ /VEGF-A pathway in colorectal cancer. Evid Based Complement Alternat Med 2015: 454279, 2015.

36. Shen A, Lin W, Chen Y, Liu L, Chen H, Zhuang Q, Lin J, Sferra TJ and Peng J: Pien Tze Huang inhibits metastasis of human colorectal carcinoma cells via modulation of TGF- $\beta 1 / \mathrm{ZEB} / \mathrm{miR}-200$ signaling network. Int J Oncol 46: 685-690, 2015
37. Wei L, Chen P, Chen Y, Shen A, Chen H, Lin W, Hong Z, Sferra TJ and Peng J: Pien Tze Huang suppresses the stem-like side population in colorectal cancer cells. Mol Med Rep 9: 261-266, 2014.

38. Siegel R, Desantis C and Jemal A: Colorectal cancer statistics, 2014. CA Cancer J Clin 64: 104-117, 2014.

39. Markowitz SD and Bertagnolli MM: Molecular origins of cancer: Molecular basis of colorectal cancer. N Engl J Med 361: 2449-2460, 2009.

40. Longley DB, Allen WL and Johnston PG: Drug resistance, predictive markers and pharmacogenomics in colorectal cancer. Biochim Biophys Acta 1766: 184-196, 2006.

41. Golebiewska A, Brons NH, Bjerkvig R and Niclou SP: Critical appraisal of the side population assay in stem cell and cancer stem cell research. Cell Stem Cell 8: 136-147, 2011.

42. Scharenberg CW, Harkey MA and Torok-Storb B: The ABCG2 transporter is an efficient Hoechst 33342 efflux pump and is preferentially expressed by immature human hematopoietic progenitors. Blood 99: 507-512, 2002.

43. Nicolis SK: Cancer stem cells and 'stemness' genes in neuro-oncology. Neurobiol Dis 25: 217-229, 2007.

44. Pastrana E, Silva-Vargas V and Doetsch F: Eyes wide open: A critical review of sphere-formation as an assay for stem cells. Cell Stem Cell 8: 486-498, 2011. 